\title{
PROJECTION SOLUTIONS OF FROBENIUS-PERRON OPERATOR EQUATIONS
}

\author{
JIU DING and TIEN TIEN LI \\ Department of Mathematics \\ University of Southern Mississippi \\ Hattiesburg, MS 39406 \\ Department of Mathematics \\ Michigan State University \\ East Lansing, MI 48824
}

(Received December 10, 1990 and in revised form June 2, 1991)

\begin{abstract}
We construct in this paper the first order and second order piecewise polynomial finite approximation schemes for the computation of invariant measures of a class of nonsingular measurable transformations on the unit interval of the real axis. These schemes are based on the Galerkin's projection method for $L^{1}$-spaces and are proved to be convergent for the class of Frobenius-Perron operators.
\end{abstract}

KEY WORDS AND PHRASES. Projection method, Frobenius-Perron operator. 1991 AMS SUBJECT CLASSIFICATION CODES. 41A15, $65 \mathrm{D} 90$.

\section{INTRODUCTION.}

Many problems in physics and engineering are concerned with the mathematical problem of existence and computation of invariant measures of measurable transformations on measure spaces [2]. For a class of nonsingular measurable transformations from $[0,1]$ into itself. Lasota and Yorke [3] established the existence of the invariant measures. Specifically, if $S:[0,1] \rightarrow[0,1]$ is a piecewise $C^{2}$ stretching mapping, then the "time average" $\frac{1}{n} \sum_{k=0}^{n=1} P^{k} f$ converges strongly in $L^{1}(0,1)$ to some $f^{*}$ of bounded variation with $P f^{*}=f^{*}$ for any density function $f \in L^{1}(0,1)$. Here $P$ is the FrobeniusPerron operator associated with $S$. In [5], $\mathrm{Li}$ and Yorke gave a sufficient condition for the uniqueness of this invariant density and thus the ergodicity of the mapping.

A straightforward numerical way to calculate the invariant measures can be obtained from the classical Birkhoff's Individual Ergodic Theorem which uses the Koopman operator instead of the Frobenius-Perron operator. By Birkhoff's theorem, if $\mu$ is an ergodic invariant probability measure under $S$, then for any measurable set $A \subset[0,1]$, the time average

$$
\lim _{n \rightarrow \infty} \frac{1}{n} \sum_{k=0}^{n-1} \chi_{A}\left(S^{k}(x)\right)
$$

which measures the "average time" spent in $A$ under iteration of $S$, exists and is $\mu(A)$ for $\mu$-almost all $x$. Here $\chi_{A}$ is the characteristic function of $A(=1$ on $A$ and $=0$ off $A$ ). Hence, one might choose almost any $x$ in $[0,1]$ and calculate the average time of the iterations $S^{k}(x)$ recurring in $A$ to obtain $\mu(A)$. However, computer round-off error can completely dominate the calculation and make the 
implementation difficult. A typical example is given in [4]; and for the purpose of overcoming this difficulty, $\mathrm{Li}$ proposed in [4] a rigorous numerical procedure which can be implemented on a computer with negligible round-off error problem. The piecewise constant approximation is used to reduce the original infinite-dimensional fixed point problem to the fixed point problems of stochastic matrices, thus solving a conjecture of Ulam's [8].

The numerical procedure proposed by $\mathrm{Li}$ has two features. The first one is that it actually belongs to the category of Galerkin's projection method, although originally it was obtained by the probability analysis. The second one is that it uses a sequence of finite rank Markov operators to approximate the Frobenius-Perron operator. Since Frobenius-Perron operators are also Markov operators, this kind of approach is natural from the viewpoint of both theory and practice. In this paper, we propose the first order and second order piecewise polynomial approximation schemes for the computation of fixed points of Frobenius-Perron operators, based on the Galerkin's projection method. The numerical experiments of Kohda and Murao [1] based on a general piecewise polynomial Galerkin approximation scheme show that the first order and second order piecewise polynomial approximation methods are much more efficient than the piecewise constant approximation in [4]. But in order to prove the convergence of their methods, the boundedness as well as the uniqueness of the invariant density are assumed which makes the convergence analysis much easier. In [1] the convergence theorem is stated and its proof is based on the variation analysis of best piecewise polynomial approximations to functions of bounded variation under the $L^{2}$-norm along with the fact that every orthogonal projection in a Hilbert space has the operator norm 1. Without the assumption of boundedness of invariant densities, we show in this paper with different approach that our scheme is convergent in $L^{1}$, which is not a Hilbert space, for the class of nonsingular measurable transformations satisfying the condition of the Lasota-Yorke theorem. The method of finite Markov approximations will be developed in further studies.

The paper is organized as follows. In Section 2, we outline some background material. Sections 3 and 4 are devoted to the first order and second order piecewise polynomial projection approximation methods and their convergence analysis, respectively.

\section{FROBENIUS-PERRON OPERATORS AND PROJECTION METHOD.}

Let $I=[0,1]$ and $S$ be a transformation from $I$ into itself. For $A \subset[0,1]$, we write $S^{-1}(A$ : for $\{x: S(x) \in A\}$. The Lebesgue measure on $[0,1]$ will be denoted by $m$. Denote by $L^{1}(0,1)$ the spac1 of all integrable functions defined on $[0,1]$ with respect to the Lebesgue measure $m . L^{1}(0,1)$ is a Banach space with norm $\|f\|=\int_{0}^{1}|f(x)| d x$. Let $S:[0,1] \rightarrow[0,1]$ be a nonsingular measurable transformation, i.e., for any measurable subset $A$ of $[0,1]$ with $m(A)=0$, we have $m\left(S^{-1}(A)\right)=0$.

The operators $P_{S}: L^{1}(0,1) \rightarrow L^{1}(0,1)$ defined by

$$
\int_{A}\left(P_{S} f\right)(x) d x=\int_{S^{-1}(A)} f(x) d x
$$

is called the Frobenius-Perron operator associated with $S$. If there is no ambiguity, we shall write $P$ for $P_{S}$. By Radon-Nikodym Theorem, the Frobenius-Perron operator is well defined when $S$ is nonsingular [2]. For $f \in L^{1}(0,1)$, the measure $\mu(A) \equiv \mu_{f}(A)=\int_{A} f(x) d x$ is invariant under $S$ if and only if $P_{S} f=f$. Here the invariance of measure $\mu$ (under $S$ ) means $\mu\left(S^{-1}(A)\right)=\mu(A)$ for every measurable set $A$. Thus, to calculate the invariant measure for $S$, we may calculate instead the fixed point of the Frobenius-Perron operator. More precisely, we need $f \in L^{1}(0,1)$ which satisfies $P_{S} f=f$. 
We list some basic properties of Frobenius-Perron operator $P_{S}$ without proof. For more detailed discussion of $P_{S}$, see [2].

(1) $P_{S}$ is a Markov operator, i.e., $P_{S}$ is linear and maps (positive) density functions to (positive) density functions. Thus $\left\|P_{S}\right\|=1$.

(2) For the $n$-th iterate $S^{n}, P_{S^{n}}=\left(P_{S}\right)^{n}$.

We give here a brief description of Galerkin's projection method in Banach spaces (see [6] for more details). Let $X$ be a Banach space. Suppose $M$ and $N$ are both closed subspaces of $X$. If $X=M+N$ and $M \cap N=\{0\}$, then we say $X$ is a direct sum of $M$ and $N$ and $M$ and $N$ are complementary to each other. In this case, we may define a linear operator $Q: X \rightarrow X$ as follows,

$$
Q x=u \text { if } x=u+v, u \in M, v \in N .
$$

This operator is continuous and satisfies $Q^{2}=Q$. We call $Q$ the projection of $X$ onto $M$ along $N$.

Now, let $X$ and $Y$ be two Banach spaces, $T: X \rightarrow Y$ be a bounded linear operator from $X$ to $Y$, and $y \in Y$. We want to solve the operator equation

$$
T x=y
$$

The general idea of projection method is as follows. Choose two sequences of finite-dimensional subspaces $X_{n}$ and $Y_{n}$ of $X$ and $Y$, respectively. Let $\left\{Q_{n}\right\}$ be a sequence of projections from $Y$ to $Y_{n}$. In $X_{n}$ we want to find $x^{(n)}$ such that $Q_{n}\left(T x^{(n)}-y\right)=0$, or

$$
Q_{n} T x^{(n)}=Q_{n} y .
$$

If we choose a basis of $X_{n}$ and a basis of $Y_{n}$, then the above approximate operator equation of finite rank can be written as a system of algebraic equations. Thus we can use the usual numerical methods to solve the linear equation and obtain the approximate solutions to the original problem. This numerical procedure is referred to as the projection method. In particular, if $X=Y$ and if we choose $X_{n}=Y_{n}$ and the same basis in $Y_{n}$ as in $X_{n}$, then the corresponding projection method is called the Galerkin's method.

\section{PIECEWISE LINEAR PROJECTION APPROXIMATION OF FROBENIUS-PERRON OPERATOR.}

A transformation $S:[0,1] \rightarrow[0,1]$ will be called piecewise $C^{2}$, if there exists a partition $0=a_{0}<a_{1}<\cdots<a_{r}=1$ of the unit interval such that for each integer $k=1, \ldots, r$, the restriction $S_{k}$ of $S$ on the open interval $\left(a_{k-1}, a_{k}\right)$ is a $C^{2}$-function which can be extended to the closed interval $\left[a_{k-1}, a_{k}\right]$ as a $C^{2}$-function. $S$ need not be continuous at the point $a_{k}$.

Assume $S:[0,1] \rightarrow[0,1]$ satisfies the condition of Lasota-Yorke theorem. That is, $S$ is piecewise $C^{2}$ satisfying in $\left|S^{\prime}(x)\right|>1$. In this section, we look for the approximate solutions of Frobenius-Perron operator equation $P_{S} f=f$ in the space of piecewise linear functions.

Divide $I=[0,1]$ into $n$ subintervals $I_{1}, I_{2}, \ldots I_{n}$. For $i=1, \ldots, n$, let $I_{i}=\left[x_{i-1}, x_{i}\right]$ and $1_{i}=\chi_{I_{i}} / m\left(I_{i}\right)$. Denote by $\Delta_{n}$ the $2 n$-dimensional subspace of $L^{1}(0,1)$ spanned by the basis $\left\{1_{i}, x 1_{i}\right\}_{i=1}^{n}$, i.e., $\Delta_{n} \subset L^{1}(0,1)$ is the set of all functions which are linear on each subinterval $I_{i}$.

Define $Q_{n}: L^{1}(0,1) \rightarrow \Delta_{n}$ by requiring that for $i=1, \ldots, n$,

$$
<f-Q_{n} f, 1_{\imath}>=0
$$

and

$$
<f-Q_{n} f, x 1_{\imath}>=0 .
$$


Here for $g \in L^{1}(0,1)$ and $h \in L^{\infty}(0,1)=\left[L^{1}(0,1)\right]^{*},\langle g, h\rangle=\int_{0}^{1} g(x) h(x) d x$. The following lemma shows that these requirements uniquely defines $Q_{n}$ and implies that $Q_{n}$ is a projection from $L^{1}(0,1)$ to $\Delta_{n}$ along ${ }^{\perp} \Delta_{n} \equiv\left\{g \in L^{1}(0,1):\langle g, h\rangle=0\right.$ for all $\left.h \in \Delta_{n}\right\}$. Because of the similarity in the "orthogonality condition" with the $L^{2}$-space case, we may call $Q_{n}: L^{1}(0,1) \rightarrow \Delta_{n}$ the orthogonal projection, even though its norm may not be 1 .

LEMMA 3.1. Let $\tilde{x}_{1}=\left(x_{i-1}+x_{1}\right) / 2, i=1, \ldots, n$. For any $f \in L^{1}(0,1)$, we have

$$
Q_{n} f=\sum_{i=1}^{n}\left(c_{1}+d_{i} x\right) 1
$$

where for $i=1, \ldots, n$,

$$
\left\{\begin{array}{l}
c_{i}=\int_{I_{i}} f(x) d x-\frac{12 \tilde{x}_{i}}{m\left(I_{i}\right)^{2}} \int_{I_{i}}\left(x-\tilde{x}_{i}\right) f(x) d x \\
d_{1}=\frac{12}{m\left(I_{i}\right)^{2}} \int_{I_{i}}\left(x-\tilde{x}_{i}\right) f(x) d x
\end{array}\right.
$$

PROOF. Let $Q_{n} f=\sum_{i=1}^{n}\left(c_{i}+d_{i} x\right) 1_{i}$, then

$$
\begin{aligned}
& \left\langle Q_{n} f, 1_{i}>=c_{i}<1_{i}, 1_{i}>+d_{i}<x 1_{i}, 1_{i}>=\frac{1}{m\left(I_{i}\right)} c_{i}+\frac{\tilde{x}_{i}}{m\left(I_{i}\right)} d_{i}\right. \\
& \left\langle Q_{n} f, x 1_{i}\right\rangle=c_{i}\left\langle 1_{i}, x 1_{i}\right\rangle+d_{i}\left\langle x 1_{i}, x 1_{i}\right\rangle \\
& =\frac{\tilde{x}_{i}}{m\left(I_{i}\right)} c_{i}+\frac{x_{i}^{2}+x_{i} x_{i-1}+x_{i-1}^{2}}{3 m\left(I_{i}\right)} d_{i} .
\end{aligned}
$$

From the condition of the orthogonal projection, we have

$$
\left\{\begin{array}{l}
\frac{1}{m\left(I_{i}\right)} c_{i}+\frac{\tilde{x}_{i}}{m\left(I_{i}\right)} d_{i}=\frac{1}{m\left(I_{i}\right)} \int_{I_{i}} f(x) d x \\
\frac{\tilde{x}_{i}}{m\left(I_{i}\right)} c_{i}+\frac{x_{i}^{2}+x_{i} x_{i}-1+x_{i-1}^{2}}{3 m\left(I_{i}\right)} d_{i}=\frac{1}{m\left(I_{i}\right)} \int_{I_{i}} x f(x) d x .
\end{array}\right.
$$

The equation (2) has a unique solution

$$
\left\{\begin{array}{l}
c_{i}=\int_{I_{i}} f(x) d x-\frac{12 \tilde{x}_{i}}{m\left(I_{i}\right)^{2}} \int_{I_{i}}\left(x-\tilde{x}_{i}\right) f(x) d x \\
d_{i}=\frac{12}{m\left(I_{i}\right)^{2}} \int_{I_{i}}\left(x-\tilde{x}_{i}\right) f(x) d x
\end{array}\right.
$$

The next lemma establishes the uniform boundedness of the sequence $Q_{n}$.

LEMMA 3.2. For all $n,\left\|Q_{n}\right\| \leq 2$.

PROOF. Given $n$ and $f \in L^{1}(0,1)$,

$$
\begin{aligned}
\left\|Q_{n} f\right\| & =\int_{0}^{1}\left|\left(Q_{n} f\right)(x)\right| d x=\int_{0}^{1} \sum_{i=1}^{n}\left|\left(c_{i}+d_{i} x\right) 1_{i}(x)\right| d x \\
& =\sum_{i=1}^{n} \int I_{i} \frac{1}{m\left(I_{i}\right)}\left|c_{i}+d_{i} x\right| d x .
\end{aligned}
$$

By (1), on the subinterval $I_{v}, Q_{n} f$ only depends on the value of $f$ on $I_{i}$. Hence it is enough to estimate one of $\frac{1}{m\left(I_{i}\right)} \int_{I_{i}}\left|c_{i}+d_{i} x\right| d x$. Without loss of generality, assume $d_{i} \neq 0$. For simplicity, let $I=I_{i}=[a, b], \tilde{x}=\tilde{x}_{i}, c=c_{i}, d=d_{i}$ and $f$ be defined on $I$. Let $\varphi(x)=(c+d x) / m(I)$. 
First of all, let us assume $f \geq 0$. If $\varphi \geq 0$, then from the first equality of (2),

$$
\begin{aligned}
\int_{I}|\varphi(x)| d x & =\int_{I} \varphi(x) d x=\frac{1}{m(I)} \int_{a}^{b}(c+d x) d x \\
& =\left.\frac{1}{m(I)} \frac{(c+d x)^{2}}{2 d}\right|_{a} ^{b}=\frac{1}{2 d m(I)}\left[(c+d b)^{2}-(c+d a)^{2}\right] \\
& =\frac{1}{2 d m(I)}\left[2 c d m(I)+d^{2} m(I) \cdot 2 \tilde{x}\right]=c+d \tilde{x} \\
& =\int_{I} f(x) d x=\int_{I}|f(x)| d x .
\end{aligned}
$$

If $\varphi \nsucceq 0$, then from the fact that $\varphi$ is the best approximation to $f$ among all linear functions on $[a, b]$ under $L^{2}$-norm if $f \in L^{2}(0,1)$, we see that $\varphi$ cannot be non-positive. Therefore $\varphi$ must have a zero $z=-\frac{c}{d}$ in $(a, b)$. We assume $\varphi(b)>0$ and $\varphi(a)<0$. The other case can be treated similarly. Thus we have

$$
\begin{aligned}
\int_{I}|\varphi(x)| d x & =\frac{1}{2}[(z-a)|\varphi(a)|+(b-z)|\varphi(b)|] \\
& =\frac{1}{2}\left[\left(b+\frac{c}{d}\right) \varphi(b)+\left(a+\frac{c}{d}\right) \varphi(a)\right],
\end{aligned}
$$

and,

$$
\begin{gathered}
b+\frac{c}{d}=\frac{m(I)^{2} \int_{I} f(x) d x}{12 \int_{I}(x-\tilde{x}) f(x) d x}+\frac{m(I)}{2}, \\
a+\frac{c}{d}=\frac{m(I)^{2} \int_{I} f(x) d x}{12 \int_{I}(x-\tilde{x}) f(x) d x}-\frac{m(I)}{2} \\
\varphi(b)=\frac{1}{m(I)}(c+d b)=\frac{1}{m(I)}\left[\int_{I} f(x) d x+\frac{6}{m(I)} \int_{I}(x-\tilde{x}) f(x) d x\right], \\
\varphi(b)=\frac{1}{m(I)}(c+d a)=\frac{1}{m(I)}\left[\int_{I} f(x) d x-\frac{6}{m(I)} \int_{I}(x-\tilde{x}) f(x) d x\right] .
\end{gathered}
$$

Hence,

$$
\begin{aligned}
\int_{I}|\varphi(x)| d x= & \frac{1}{2}\left\{\left(\frac{m(I)^{2} \int_{I} f(x) d x}{12 \int_{I}(x-\tilde{x}) f(x) d x}+\frac{m(I)}{2}\right)\right. \\
& \cdot \frac{1}{m(I)}\left(\int_{I} f(x) d x+\frac{6}{m(I)} \int_{I}(x-\tilde{x}) f(x) d x\right) \\
& +\left(\frac{m(I)^{2} \int_{I} f(x) d x}{12 \int_{I}(x-\tilde{x}) f(x) d x}-\frac{m(I)}{2}\right) \\
& \left.\cdot \frac{1}{m(I)}\left(\int_{I} f(x) d x-\frac{6}{m(I)} \int_{I}(x-\tilde{x}) f(x) d x\right)\right\} \\
= & \frac{m(I)\left[\int_{I} f(x) d x\right]^{2}}{12 \int_{I}(x-\tilde{x}) f(x) d x}+\frac{3}{m(I)} \int_{I}(x-\tilde{x}) f(x) d x \\
\leq & \frac{m(I)\left[\int_{I} f(x) d x\right]^{2}}{12 \int_{I}(x-\tilde{x}) f(x) d x}+\frac{3}{2} \int_{I} f(x) d x .
\end{aligned}
$$


Note that $z=-\frac{c}{d} \in(a, b)$, we have

$$
a<\tilde{x}-\frac{m(I)^{2} \int_{I} f(x) d x}{12 \int_{I}(x-\tilde{x}) f(x) d x}<b
$$

It follows that

$$
\frac{m(I)^{2} \int_{I} f(x) d x}{12 \int_{I}(x-\tilde{x}) f(x) d x}<\tilde{x}-a=\frac{m(I)}{2}
$$

Therefore

$$
\int_{I}|\varphi(x)| d x<\frac{1}{2} \int_{I} f(x) d x+\frac{3}{2} \int_{I} f(x) d x=2 \int_{I} f(x) d x
$$

For general $f \in L^{1}(I)$, write $f=f^{+}-f^{-}$where $f^{+}=\max \{f, 0\}$ and $f^{-}=\max \{-f, 0\}$, and we have

$$
\begin{aligned}
\int_{I}|\varphi(x)| d x & =\int_{I}|Q f| d x=\int_{I}\left|Q f^{+}-Q f^{-}\right| d x \\
& \leq \int_{I}\left|Q f^{+}\right| d x+\int_{I}\left|Q f^{-}\right| d x \\
& \leq 2 \int_{I} f^{+} d x+2 \int_{I} f^{-} d x=2 \int_{I}|f| d x
\end{aligned}
$$

where $Q: L^{1}(I) \rightarrow \operatorname{Span}\{1, x\}$ is the above mentioned orthogonal projection.

From the above estimate, we obtain

$$
\begin{aligned}
\left\|Q_{n} f\right\| & =\int_{0}^{1}\left|\left(Q_{n} f\right)(x)\right| d x=\sum_{i=1}^{n} \int_{I_{1}} \frac{1}{m\left(I_{i}\right)}\left|c_{i}+d_{i} x\right| d x \\
\leq & \sum_{i=1}^{n} 2 \int_{I_{i}}|f(x)| d x=2 \int_{0}^{1}|f(x)| d x=2\|f\|,
\end{aligned}
$$

i.e., for all $n,\left\|Q_{n}\right\| \leq 2$. (Q.E.D.)

LEMMA 3.3. When mesh $\left(\Delta_{n}\right) \equiv \max \left\{m\left(I_{i}\right): 1 \leq i \leq n\right\} \rightarrow 0, Q_{n} f \rightarrow f$ for all $f \in L^{1}(0,1)$.

PROOF. Given $f \in L^{1}(0,1)$ and $\varepsilon>0$, there exists a continuous function $g$ such that $\|f-g\|<\varepsilon$. Now

$$
\begin{aligned}
\left\|Q_{n} g-g\right\|= & \sum_{i=1}^{n} \int_{I_{i}}\left|\left(Q_{n} g\right)(y)-g(y)\right| d y \\
= & \sum_{i=1}^{n} \int_{I_{i}}\left|\frac{1}{m\left(I_{i}\right)}\left(c_{i}+d_{i} y\right)-g(y)\right| d y \\
= & \sum_{i=1}^{n} \int_{I_{i}} \mid \frac{1}{m\left(I_{i}\right)} \int_{I_{i}} g(x) d x-\frac{12 \tilde{x}_{i}}{m\left(I_{i}\right)^{3}} \int_{I_{i}}\left(x-\tilde{x}_{i}\right) g(x) d x \\
& \quad+\left(\frac{12}{m\left(I_{i}\right)^{3}} \int_{I_{i}}\left(x-\tilde{x}_{i}\right) g(x) d x\right) y-g(y) \mid d y
\end{aligned}
$$




$$
\begin{aligned}
& \leq \sum_{i=1}^{n} \int_{I_{i}}\left|\frac{1}{m\left(I_{i}\right)} \int_{I_{i}} g(x) d x-g(y)\right| d y \\
& \quad+\sum_{i=1}^{n} \int_{i_{i}\left|\frac{12}{m\left(I_{i}\right)^{3}}\left(\int_{I_{i}}\left(x-\tilde{x}_{i}\right) g(x) d x\right)\left(y-\tilde{x}_{i}\right)\right| d y}^{\leq} \\
& \quad \sum_{i=1}^{n} \int_{I_{i}} \frac{1}{m\left(I_{i}\right)}\left(\int_{I_{i}}|g(x)-g(y)| d x\right) d y \\
& \quad+\sum_{i=1}^{n} \frac{12}{m\left(I_{i}\right)^{3}} \int_{I_{i}}\left|\left(x-\tilde{x}_{i}\right) g(x)\right| d x \cdot \int_{I_{i}}\left|y-\tilde{x}_{i}\right| d y .
\end{aligned}
$$

Since $g$ is uniformly continuous on $[0,1]$, when mesh $\left(\Delta_{n}\right)$ is sufficiently small, for any $x, y \in I_{v}, i=1, \ldots, n$, we have $|g(x)-g(y)|<\varepsilon$. Applying Hölder's inequality, we get

$$
\begin{aligned}
\left\|Q_{n} g-g\right\| \leq & \sum_{i=1}^{n} \int_{I_{i}} \frac{1}{m\left(I_{i}\right)} \cdot m\left(I_{i}\right) \frac{\varepsilon}{2} d y \\
& +\sum_{i=1}^{n} \frac{12}{m\left(I_{i}\right)^{3}}\left[\int_{I_{i}}\left(x-\tilde{x}_{i}\right)^{2} d x\right]^{1 / 2} \\
& \cdot\left[\int_{I_{i}} g(x)^{2} d x\right]^{1 / 2} \cdot \int_{I_{i}}\left|y-\tilde{x}_{i}\right| d y \\
& \leq \sum_{i=1}^{n} \frac{\varepsilon}{2} m\left(I_{i}\right)+\sum_{i=1}^{n} \frac{12}{m\left(I_{i}\right)^{3}}\left\{\left[\frac{\left(x-\tilde{x}_{i}\right)^{3}}{3}\right]_{x_{i}-1}^{x_{i}}\right\}^{1 / 2} \\
& \cdot\left[\int_{I_{i}} g(x)^{2} d x\right]^{1 / 2} \cdot\left[\frac{\left(m\left(I_{i}\right) / 2\right)^{2}}{2}+\frac{\left(m\left(I_{i}\right) / 2\right)^{2}}{2}\right]^{1 / 2} . \\
& =\frac{\varepsilon}{2}+\frac{\sqrt{3}}{2} \sum_{i=1}^{n} m\left(I_{i}\right)^{1 / 2}\left[\int_{I_{i}} g(x)^{2} d x\right]^{1 / 2}
\end{aligned}
$$

For $n$ sufficiently large, $\left(\int_{I_{1}} g(x)^{2} d x\right)^{1 / 2}<\frac{\varepsilon}{\sqrt{3}}, i=1, \ldots, n$, hence,

$$
\left\|Q_{n} g-g\right\| \leq \frac{\varepsilon}{2}+\frac{\varepsilon}{2} \sum_{i=1}^{n} m\left(I_{i}\right)^{1 / 2} \leq \frac{\varepsilon}{2}+\frac{\varepsilon}{2}=\varepsilon .
$$

Together with Lemma 3.2 , for $n$ sufficiently large,

$$
\begin{aligned}
\left\|Q_{n} f-f\right\| & \leq\left\|Q_{n} f-Q_{n} g\right\|+\left\|Q_{n} g-g\right\|+\|g-f\| \\
& \leq 2\|f-g\|+\varepsilon+\|f-g\| \leq 4 \varepsilon .
\end{aligned}
$$

This proves $\lim _{n \rightarrow \infty} Q_{n} f=f$. (Q.E.D.)

The following result is the key to our convergence analysis.

LEMMA 3.4. For any $f \in L^{1}(0,1)$ of bounded variation and for all $n$

$$
\stackrel{1}{\vee} Q_{n} f \leq 13 \underset{0}{\stackrel{1}{\vee}} f
$$

where $v_{0}^{1} f$ is the variation of $f$ on $[0,1]$ (for the definition of variation, see [7]). 
472

J. DING AND TIT. LI

PROOF. By definition, $Q_{n} f=\sum_{i=1}^{n}\left(c_{3}+d_{i} x\right) 1_{1}$, where $\left\{c_{2}, d_{3}\right\}$ are given by (1). Since $Q_{n} f$ is piecewise linear, its variation is given by

$$
\begin{aligned}
& \underset{0}{1} Q_{n} f=\sum_{i=1}^{n} 1 \frac{1}{m\left(I_{i}\right)}\left|\left(c_{i}+d_{i} x_{i}\right)-\left(c_{i}+d_{i} x_{i-1}\right)\right| \\
& +\sum_{i=1}^{n-1}\left|\frac{c_{i}+d_{1} x_{i}}{m\left(I_{i}\right)}-\frac{c_{i+1}+d_{i+1} x_{i}}{m\left(I_{1}+1\right)}\right| \\
& =\sum_{i=1}^{n}\left|d_{1}\right|+\sum_{i=1}^{n-1}\left|\frac{c_{i}}{m\left(I_{i}\right)}-\frac{c_{i+1}}{m\left(I_{1}+1\right)}+\left(\frac{d_{i}}{m\left(I_{i}\right)}-\frac{d_{i+1}}{m\left(I_{i+1}\right)}\right) x_{i}\right| \\
& =\sum_{i=1}^{n}\left|d_{i}\right|+\sum_{i=1}^{n-1} \mid \frac{1}{m\left(I_{i}\right)} \int_{I_{i}} f(x) d x-\frac{1}{m\left(I_{i+1}\right)} \int_{I_{i+1}} f(x) d x \\
& +\frac{12 \tilde{x}_{i}}{m\left(I_{i+1}\right)^{3}} \int_{I_{i+1}}\left(x-\tilde{x}_{i+1}\right) f(x) d x-\frac{12 \tilde{x}_{i}}{m\left(I_{i}\right)^{3}} \int_{I_{i}}\left(x-\tilde{x}_{i}\right) f(x) d x \\
& +\frac{12 x_{i}}{m\left(I_{i}\right)^{3}} \int_{I_{i}}\left(x-\tilde{x}_{i}\right) f(x) d x-\frac{12 x_{i}}{m\left(I_{i+1}\right)^{3}} \int_{I_{i+1}}\left(x-\tilde{x}_{i+1}\right) f(x) d x \mid \\
& =\sum_{i=1}^{n}\left|d_{i}\right|+\sum_{i=1}^{n-1} \mid \frac{1}{m\left(I_{i}\right)} \int_{I_{i}} f(x) d x-\frac{1}{m\left(I_{i+1}\right)} \int_{I_{i+1}} f(x) d x \\
& +\frac{12\left(\tilde{x}_{i+1}-x_{i}\right)}{m\left(I_{i+1}\right)^{3}} \int_{I_{i+1}}\left(x-\tilde{x}_{i+1}\right) f(x) d x \\
& +\frac{12\left(x_{i}-\tilde{x}_{i}\right)}{m\left(I_{i}\right)^{3}} \int_{I_{i}}\left(x-\tilde{x}_{i}\right) f(x) d x \\
& =\sum_{i=1}^{n}\left|d_{i}\right|+\sum_{i=1}^{n-1} \mid \frac{1}{m\left(I_{i}\right)} \int_{I_{i}} f(x) d x-\frac{1}{m\left(I_{i+1}\right)} \int_{I_{i+1}} f(x) d x \\
& +\frac{6}{m\left(I_{i+1}\right)^{2}} \int_{I_{i+1}}\left(x-\tilde{x}_{i+1}\right) f(x) d x+\frac{6}{m\left(I_{i}\right)^{2}} \int_{I_{i}}\left(x-\tilde{x}_{i}\right) f(x) d x
\end{aligned}
$$

From the definition of $d_{i}$ we have

$$
\begin{aligned}
\underset{0}{1} Q_{n} f \leq & \sum_{i=1}^{n}\left|d_{i}\right|+\sum_{i=1}^{n-1}\left|\frac{1}{m\left(I_{i}\right)} \int_{I_{i}} f(x) d x-\frac{1}{m\left(I_{i+1}\right)} \int_{I_{i+1}} f(x) d x\right| \\
& +\sum_{i=1}^{n-1}\left|\frac{1}{2} d_{i+1}+\frac{1}{2} d_{i}\right| \\
\leq & \sum_{i=1}^{n}\left|d_{i}\right|+\sum_{i=1}^{n-1}\left|\frac{1}{m\left(I_{i}\right)} \int_{I_{i}} f(x) d x-\frac{1}{m\left(I_{i+1}\right)} \int_{I_{i+1}} f(x) d x\right| \\
& +\sum_{i=1}^{n}\left|d_{i}\right| .
\end{aligned}
$$


It is easy to see that the middle summation of the above inequality is not greater than $\vee_{0}^{1} f$ (for a proof, see [4]). Hence,

$$
\underset{0}{1} Q_{n} f \leq 2 \sum_{i=1}^{n}\left|d_{i}\right|+\underset{0}{1} f
$$

Now we estimate $\sum_{i=1}^{n}\left|d_{1}\right|$. Let $F_{1}(x)=\int_{x_{1}-1}^{x} f(t) d t$, then the formula of integration by parts for the Stieljes-Lebesgue integral [6] gives

$$
\begin{aligned}
d_{i} & =\frac{12}{m\left(I_{i}\right)^{2}} \int_{I_{i}}\left(x-\tilde{x}_{i}\right) f(x) d x=\frac{12}{m\left(I_{i}\right)^{2}} \int_{I_{i}}\left(x-\tilde{x}_{i}\right) d F_{i}(x) \\
& =\frac{12}{m\left(I_{i}\right)^{2}}\left[\left.\left(x-\tilde{x}_{i}\right) F_{i}(x)\right|_{x_{i-1}} ^{x_{i}}-\int_{I_{i}} F_{i}(x) d\left(x-\tilde{x}_{i}\right)\right] \\
& =\frac{12}{m\left(I_{i}\right)^{2}}\left[\frac{m\left(I_{i}\right)}{2} F_{i}\left(x_{i}\right)-\int_{I_{i}} F_{i}(x) d x\right] \\
& =\frac{6}{m\left(I_{i}\right)} \int I_{i} f(t) d t-\frac{12}{m\left(I_{i}\right)^{2}} \iint_{I_{i}}\left(\int_{x_{i-1}}^{x} f(t) d t\right) d x \\
& =6\left[\frac{1}{m\left(I_{i}\right)} \int_{I_{i}} f(t) d t-\frac{1}{A_{i}} \iint_{\Omega_{i}} f(t) d t d x\right]
\end{aligned}
$$

where $\Omega_{1}=\left\{(x, t): x_{i-1} \leq x \leq x_{i}, x_{i-1} \leq t \leq x\right\}$ is a triangular region in the $(x, t)$-plane and $A_{t}=\frac{1}{2} m\left(I_{t}\right)^{2}$ is the area of $\Omega_{i}$. With the same reason described in [4], we obtain

$$
\sum_{i=1}^{n}\left|d_{i}\right|=6 \sum_{i=1}^{n}\left|\frac{1}{m\left(I_{i}\right)} \int_{I_{i}} f(t) d t-\frac{1}{A_{i}} \iint_{\Omega_{i}} f(t) d t d x\right| \leq 6 \underset{0}{\mathbb{V}} f
$$

Therefore,

$$
\stackrel{1}{\vee} Q_{n} f \leq 13 \underset{0}{\stackrel{1}{v} f}
$$

Let $P_{n}=\left.Q_{n} \circ P_{S}\right|_{\Delta_{n}}$, where $\left.P_{S}\right|_{\Delta_{n}}$ is the restriction of $P_{S}$ on $\Delta_{n}$, then $P_{n}: \Delta_{n} \rightarrow \Delta_{n}$ is linear. We want to find the fixed points of $P_{n}$ in $\Delta_{n}$. For this purpose, we first investigate the representation of $P_{n}$ under the basis $\left\{1_{i}, x 1_{i}\right\}_{i=1}^{n}$.

LEMMA 3.5. For $i=1, \ldots, n$,

$$
\begin{gathered}
P_{n} 1_{\imath}=\sum_{j=1}^{n} c_{j}\left(1_{i}\right) 1_{j}+\sum_{j=1}^{n} d_{j}\left(1_{i}\right) x 1_{j} \\
P_{n}\left(x 1_{i}\right)=\sum_{j=1}^{n} c_{j}\left(x 1_{i}\right) 1_{j}+\sum_{j=1}^{n} d_{j}\left(x 1_{i}\right) x 1_{j},
\end{gathered}
$$

where

$$
\begin{aligned}
& c_{j}\left(1_{\imath}\right)=\frac{m\left(S^{-1}\left(I_{j}\right) \cap I_{\imath}\right)}{m\left(I_{i}\right)}-\frac{12 \tilde{x}_{j}}{m\left(I_{j}\right)^{2}} \int_{I_{j}}\left(x-\tilde{x}_{\jmath}\right)\left(P 1_{i}(x) d x\right. \\
& d_{j}\left(1_{i}\right)=\frac{12}{m\left(I_{j}\right)^{2}} \int_{I_{j}}\left(x-\tilde{x}_{j}\right)\left(P 1_{i}\right)(x) d x
\end{aligned}
$$




$$
\begin{aligned}
& c_{\jmath}\left(x 1_{\imath}\right)=\int_{I_{j}}\left(P\left(x 1_{\imath}\right)\right)(x) d x-\frac{12 \tilde{x}_{j}}{m\left(I_{\jmath}\right)^{2}} \int_{I_{j}}\left(x-\tilde{x}_{\jmath}\right)\left(P\left(x 1_{i}\right)\right)(x) d x, \\
& d_{\jmath}\left(x 1_{\mathfrak{a}}\right)=\frac{12}{m\left(I_{\jmath}\right)^{2}} \int_{I_{j}}\left(x-\tilde{x}_{\jmath}\right)\left(P\left(x 1_{\imath}\right)\right)(x) d x .
\end{aligned}
$$

PROOF. By definition, $P_{n} 1_{1}=Q_{n} \circ P_{S} 1_{v}, P_{n}\left(x 1_{8}\right)=Q_{n} \circ P_{S}\left(x 1_{8}\right)$. From the definition of $P_{S}, \int_{I_{3}}\left(P_{S} 1_{3}\right)(x) d x=\frac{m\left(S^{-1}\left(I_{j}\right) \cap I_{t}\right)}{m\left(I_{a}\right)}$. Combined with Lemma 3.1, we have the result. (Q.E.D.)

LEMMA 3.6. $P_{n}$ has a nontrivial fixed point $f_{n}$ in $\Delta_{n}$.

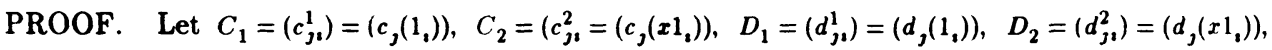

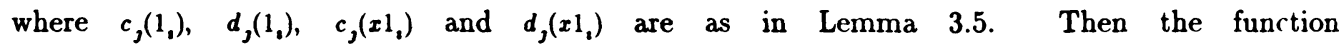
$f_{n}(x)=\sum_{1=1}^{n} c_{1} 1_{1}+\sum_{i=1}^{n} d_{1} x 1_{1}$, is a fixed point of $P_{n}$ if and only if the column vector $\left(c_{1} \ldots, c_{n}, d_{1}, \ldots, d_{n}\right)^{T}$ is a fixed point of the matrix

$$
\tilde{P}_{n}=\left[\begin{array}{ll}
C_{1} & C_{2} \\
D_{1} & D_{2}
\end{array}\right] .
$$

We first prove that the row vector $l=\left(1, \ldots 1, \tilde{x}_{1}, \ldots, \tilde{x}_{n}\right)$ satisfies $l=l \tilde{P}_{n}$. In fact, from the first equality of (2),

$$
\begin{aligned}
\sum_{j=1}^{n}\left(c_{j}\left(1_{i}\right)+\tilde{x}, d,\left(1_{i}\right)\right) & =\sum_{j=1}^{n} \int_{I_{j}}\left(P 1_{i}\right)(x) d x=\sum_{j=1}^{n} \int s^{-1}\left(I_{j}, 1_{i}(x) d x\right. \\
& =\sum_{j=1}^{n} \frac{m\left(S^{-1}\left(I_{j}\right) \cap I_{i}\right)}{m\left(I_{i}\right)}=1 \\
\sum_{j=1}^{n}\left(c,\left(x 1_{i}\right)+\tilde{x}_{j} d_{j}\left(x 1_{i}\right)\right) & =\sum_{j=1}^{n} \int_{I_{j}}\left(P\left(x 1_{i}\right)\right)(x) d x \\
& =\sum_{j=1}^{n} \int S^{\left.-1_{(I}\right)} x 1_{i}(x) d x=\int_{0}^{1} x 1_{i}(x) d x \\
& =\frac{1}{m\left(I_{i}\right)} \int I_{i} x d x=\frac{1}{m\left(I_{i}\right)} \frac{x_{i}^{2}-x_{i-1}^{2}}{2}=\tilde{x}_{i} .
\end{aligned}
$$

Hence the matrix $\tilde{P}_{n}$ has eigenvalue 1 and it follows that $\tilde{P}_{n} \mu=\mu$ has a nontrivial solution. (Q.E.D.)

In [3], Lasota and Yorke prove that, if $S:[0,1] \rightarrow[0,1]$ is a piecewise $C^{2}$-function satisfying $M=$ inf $|S|>2$, then for any $f \in L^{1}(0,1)$ of bounded variation, $\vee_{0}^{1} P_{S} f \leq \alpha\|f\|+\beta \vee_{0}^{1} f$ with $\alpha>0$ and $\beta=\frac{2}{M}<1$. We shall call this inequality the Lasota-Yorke inequality. With this result, we can prove the following

LEMMA 3.7. Suppose $S:[0,1] \rightarrow[0,1]$ is piecewise $C^{2}$ and $M \equiv$ inf $\left|S^{\prime}\right|>26$. Then the sequence $\left\{\vee_{0}^{1} f_{n}\right\}$ is bounded with $f_{n}$ the fixed points of $P_{n}$ satisfying $\left\|f_{n}\right\|=1$.

PROOF. Since $f_{n}$ is piecewise linear, it has bounded variation. From the Lasota-Yorke inequality, $P f_{n}$ is a function of bounded variation. From the same inequality and the fact that $f_{n}=P_{n} f_{n}=Q_{n} \circ P f_{n}$, using Lemma 3.4, we obtain

$$
\begin{aligned}
& \underset{0}{\stackrel{1}{\vee}} f_{n}=\underset{0}{\stackrel{1}{V}} Q_{n} \circ P f_{n} \leq 13 \underset{0}{\stackrel{1}{\vee}} P f_{n} \leq 13\left(\alpha\left\|f_{n}\right\|+\beta \underset{0}{\left.\stackrel{1}{\vee} f_{n}\right)}\right. \\
& =13 \alpha+13 \beta \underset{0}{\stackrel{1}{\vee}} f_{n}=13 \alpha+\frac{26}{M} \underset{0}{1} f_{n} .
\end{aligned}
$$


By assumption, $M>26$, therefore for all $n$

$$
\stackrel{1}{\vee} f_{n} \leq \frac{13 \alpha}{1-26 / M}<\infty
$$

Now we can prove our convergence theorem for the first-order piecewise polynomial Galerkin approximation scheme for Frobenius-Perron operator equations.

THEOREM 3.1. Suppose $S:[0,1] \rightarrow[0,1]$ is piecewise $C^{2}$ and $M=$ inf $\left|S^{\prime}\right|>26$. Then for any $n$, $P_{n}$ has a fixed point $f_{n}$ with $\left\|f_{n}\right\|=1$ in $\Delta_{n}$ and when mesh $\left(\Delta_{n}\right) \rightarrow 0$, there exists a subsequence $\left\{f_{n_{1}}\right\} \subset\left\{f_{n}\right\}$ such that $f_{n_{i}}$ converges to a fixed point of $P_{S}$.

PROOF. By Lemma 3.7 and the Helly Theorem [7], there is a subsequence $\left\{f_{n_{1}}\right\} \subset\left\{f_{n}\right\}$ which converges to some $f \in L^{1}(0,1)$. Now

$$
\begin{aligned}
\left\|P_{S} f-f\right\| \leq \| f & -f_{n_{i}}\|+\| f_{n_{i}}-Q_{n_{i}} \circ P_{S} f_{n_{i}} \| \\
& +\left\|Q_{n_{i}} \circ P_{S} f_{n_{i}}-Q_{n_{i}} \circ P_{S} f\right\|+\left\|Q_{n_{i}} \circ P_{S} f-P_{S} f\right\| .
\end{aligned}
$$

Since $\left\{\left\|Q_{n_{i}} \circ P_{S}\right\|\right\}$ is uniformly bounded and $Q_{n_{i}} \circ P_{S} f_{n_{i}}=f_{n_{i}}$, Lemma 3.3 implies that the right hand side of the above inequality approaches zero as $i \rightarrow \infty$. Thus $P_{S} f=f$. (Q.E.D.)

COROLLARY 3.1. Suppose $S:[0,1] \rightarrow[0,1]$ is piecewise $C^{2}$ and $M=$ inf $|S|>1$, then a sequence of functions can be constructed from piecewise linear functions which converge to a fixed point of $P_{S}$.

PROOF. Choose $k>0$ such that $M^{k}>26$. Let $\varphi=S^{k}$. Then $P_{n}(\varphi)$ has a fixed point $f_{n}^{(\varphi)}$ of unit length in $\Delta_{n}$. Define

$$
g_{i}=\frac{1}{k} \sum_{j=0}^{k-1}\left(P_{S}\right)^{i} f_{n_{i}}^{(\varphi)},
$$

where $f_{n_{i}}$ is a convergent subsequence of $\left\{f_{n}\right\}$ from the above theorem. Then $g_{i}$ converges, by Theorem 3.1, to

$$
g=\frac{1}{k} \sum_{j=1}^{k-1}\left(P_{S}\right)^{\jmath} f^{(\varphi)}
$$

where $f^{(\varphi)}$ is a fixed point of $\varphi=S^{k}$. This $g$ is a fixed point of $P_{S}$. In fact, since $\left(P_{S}\right)^{k} f^{(\varphi)}=P_{S^{k}}$ $f^{(\varphi)}=P_{\varphi} f^{(\varphi)}=f^{(\varphi)}$,

$$
P_{S} g=\frac{1}{k}\left\{P_{S} f^{(\varphi)}+\cdots+\left(P_{S}\right)^{k} f^{(\varphi)}\right\}=g .
$$

\section{PIECEWISE QUADRATIC PROJECTION APPROXIMATION OF FROBENIUS-PERRON OPERATOR.}

In this section, we shall generalize the piecewise linear approximation of the previous section to the piecewise quadratic one, that is, we look for the approximate solutions of Frobenius-Perron operator equation in the space of piecewise quadratic functions. Let $x_{0}=0<x_{1}<\cdots<x_{n-1}<x_{n}=1$ be a finite partition of the interval $[0,1]$ as before. For $i=1, \ldots, n, I_{i}=\left[x_{i-1}, x_{i}\right], \tilde{x}_{i}=\frac{x_{i-1}+x_{i}}{2}$. Let mesh $\left(\Delta_{n}\right)=\max m\left(I_{i}\right)$ and $\Delta_{n}=\operatorname{span}\left\{1_{i}, x 1_{i}, x^{2} 1_{i}\right\}_{i=1}^{n}$ where $1_{i}=\frac{1}{m\left(I_{i}\right)} \chi_{I_{i}} . \Delta_{n} \subset L^{1}(0,1)$ is a subspace of dimension $3 n$.

Define the projection $Q_{n}: L^{1}(0,1) \rightarrow \Delta_{n}$ by the orthogonal conditions, for $i=1, \ldots, n$

$$
\left\langle f-Q_{n} f, 1_{i}\right\rangle=0,\left\langle f-Q_{n} f, x 1_{i}\right\rangle=0,\left\langle f-Q_{n} f, x^{2} 1_{i}\right\rangle=0 .
$$


Let $Q_{n} f=\sum_{j=1}^{n}\left(c_{j}+d_{j} x+e, x^{2}\right) 1$. We show that $\left\{c_{j}, d_{j}, e,\right\}_{j=1}^{n}$ are uniquely determined by the above conditions.

For $i=1, \ldots, n$, straightforward calculation gives

$$
\begin{aligned}
& \left\langle Q_{n} f, 1_{1}\right\rangle=\frac{1}{m\left(I_{2}\right)^{2}}+\frac{\tilde{x}_{1}}{m\left(I_{1}\right)} d_{1}+\frac{x_{1}^{2}+x_{1} x_{1-1}+x_{i-1}^{2}}{3 m\left(I_{1}\right)} e_{1},
\end{aligned}
$$

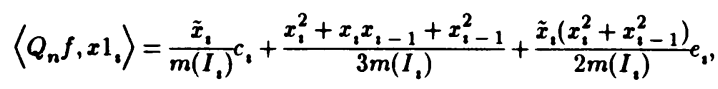

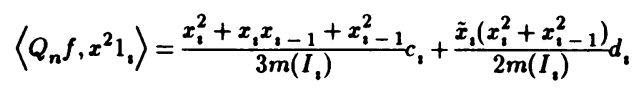

$$
\begin{aligned}
& +\frac{1}{5 m\left(I_{1}\right)}\left(x_{i}^{4}+x_{1}^{3} x_{i-1}+x_{i}^{2} x_{i-1}^{2}+x_{i-1}^{4}\right) e_{i} .
\end{aligned}
$$

By the orthogonal condition, we have the following equations

$$
\left\{\begin{array}{l}
c_{i}+\tilde{x}_{i} d_{i}+\frac{1}{3}\left(x_{i}^{2}+x_{i} x_{i-1}+x_{i-1}^{2}\right) e_{i}=\int_{I_{i}} f(x) d x \\
\tilde{x}_{i} c_{i}+\frac{1}{3}\left(x_{i}^{2}+x_{i} x_{i-1}+x_{i-1}^{2}\right) d_{i}+\frac{\tilde{x}_{i}}{2}\left(x_{i}^{2}+x_{i-1}^{2}\right)=\int_{I_{i}} x f(x) d x \\
\frac{1}{3}\left(x_{i}^{2}+x_{i} x_{i-1}+x_{i-1}^{2}\right) c_{i}+\frac{\tilde{x}_{i}}{2}\left(x_{i}^{2}+x_{i-1}^{2}\right) d_{i}+ \\
\frac{1}{5}\left(x_{1}^{4}+x_{i-1}^{3} x_{i-1}+x_{i}^{2} x_{i-1}^{2}+x_{i} x_{i-1}^{3}+x_{i-1}^{4}\right) e_{i}=\int_{I_{i}} x^{2} f(x) d x .
\end{array}\right.
$$

Eliminate $c_{\imath}$ from the above system, we have

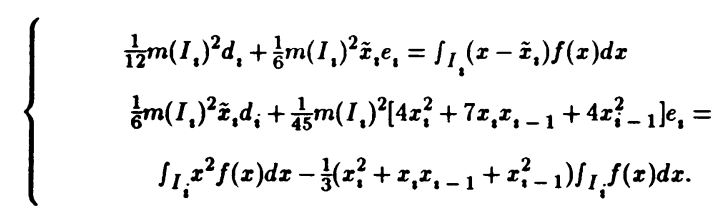

The solutions are given by

$$
\left\{\begin{array}{c}
c_{i}=\frac{3}{2} \int_{I_{i}} f(x) d x-\frac{12 \tilde{x}_{i}}{m\left(I_{i}\right)^{2}} \int_{I_{i}} x f(x) d x-\frac{3 x_{i} x_{i-1}}{m\left(I_{i}\right)^{2}} \int_{I_{i}} f(x) d x+ \\
\frac{60}{m\left(I_{i}\right)^{4}}\left[2 \tilde{x}_{i}^{2}+x_{i} x_{i-1}\right] \int_{I_{i}}\left(x-\tilde{x}_{i}\right) f(x) d x \\
d_{i}=\frac{12}{m\left(I_{i}\right)^{2}} \int_{I_{i}} x f(x) d x+\frac{18 \tilde{x}_{i}}{m\left(I_{i}\right)^{2}} \int_{I_{i}} f(x) d x- \\
\frac{360}{m\left(I_{i}\right)^{4}} \tilde{x}_{i} \int_{I_{i}}\left(x-\tilde{x}_{i}\right)^{2} f(x) d x \\
e_{i}=\frac{180}{m\left(I_{i}\right)^{4}} \int_{I_{i}}\left[\left(x-\tilde{x}_{i}\right)^{2}-\frac{1}{12} m\left(I_{i}\right)^{2}\right] f(x) d x .
\end{array}\right.
$$

LEMMA 4.1. $\left\|Q_{n}\right\| \leq 62$ for all $n$.

PROOF. The value of $Q_{n} f$ in the subinterval $I_{z}$ depends only on the value of $f$ on $I_{z}$. So it is enough to estimate the integral $\int_{I_{i}}\left|\left(Q_{n} f\right)(x)\right| d x$. Let $I=I_{i}, I=[a, b], \tilde{x}=\frac{a+b}{2}$ and let $\varphi(x)=\frac{1}{m(I)}\left(c+d x+e x^{2}\right)$ be the orthogonal projection of $f$ to $S p a n\left\{1_{I}, x 1_{I}, x^{2} 1_{I}\right\}$. First of all, assume $f \geq 0$. We consider different cases. 
(i) $\varphi \geq 0$. Then from the first equality of (3),

$$
\begin{aligned}
\int_{I}|\varphi(x)| d x & =\int_{I} \varphi(x) d x=\frac{1}{m(I)} \int_{I}\left(c+d x+e x^{2}\right) d x \\
& =\frac{1}{m(I)}\left[c x+\frac{d}{2} x^{2}+\frac{e}{3} x^{3}\right]_{a}^{b}=c+d \tilde{x}+\frac{1}{3}\left(a^{2}+a b+b^{2}\right) e \\
& =\int_{I} f(x) d x=\int_{I}|f(x)| d x .
\end{aligned}
$$

(ii) $\varphi \geq 0$. Then $\varphi$ has distinct zeros on the real axis. Without loss of generality, we may assume $e>0$. Consider different distribution of the zeros. Let $\zeta_{1}$ and $\zeta_{2}$ be zeros of $\varphi$ with $\zeta_{1}<\zeta_{2}$.

Firstly, assume $\zeta_{1} \in(a, b)$ and $\zeta_{2} \in(a, b)$. Then $\zeta_{1}+\zeta_{2}=-\frac{d}{e}, \zeta_{1} \cdot \zeta_{2}=\frac{c}{e}$, and

$$
\begin{aligned}
\int_{I}|\varphi(x)| d x= & \frac{1}{m(I)}\left[\int_{a}^{\zeta_{1}}\left(c+d x+e x^{2}\right) d x-\int_{\zeta_{1}}^{\zeta_{2}}\left(c+d x+e x^{2}\right) d x\right. \\
& \left.\quad+\int_{\zeta_{2}}^{b}\left(c+d x+e x^{2}\right) d x\right] \\
= & \frac{1}{m(I)}\left\{\left[c x+\frac{d}{2} x^{2}+\frac{e}{3} x^{3}\right]_{a}^{\zeta_{1}}-\left[c x+\frac{d}{2} x^{2}+\frac{3}{3} x^{3}\right\}_{k_{1}}\right. \\
& \left.+\left[c x+\frac{d}{2} x^{2}+\frac{e}{3} x^{3}\right\}_{2}^{b}\right\} \\
= & \frac{1}{m(I)}\left[c(b-a)+\frac{d}{2}\left(b^{2}-a^{2}\right)+\frac{e}{3}\left(b^{3}-a^{3}\right)\right] \\
& +\frac{2}{m(I)}\left[c\left(\zeta_{1}-\zeta_{2}\right)+\frac{d}{2}\left(\zeta_{1}^{2}-\zeta_{2}^{2}\right)+\frac{e}{3}\left(\zeta_{1}^{3}-\zeta_{2}^{3}\right)\right] \\
= & {\left[c+d \tilde{x}+\frac{e}{3}\left(a^{2}+a b+b^{2}\right)\right] } \\
& -\frac{2\left(\zeta_{2}-\zeta_{1}\right)}{m(I)}\left[c+\frac{d}{2}\left(\zeta_{1}+\zeta_{2}\right)+\frac{e}{3}\left(\zeta_{1}+\zeta_{2}\right)^{2}-\zeta_{1} \zeta_{2}\right] \\
= & \int_{I} f(x) d x-\frac{2\left(\zeta_{2}-\zeta_{1}\right)}{m(I)}\left[c+\frac{d}{2}\left(-\frac{d}{e}\right)+\frac{e}{3}\left(-\frac{d}{e}\right)^{2}-\frac{c}{e}\right] \\
= & \int_{I} f(x) d x-\frac{2\left(\zeta_{2}-\zeta_{1}\right)}{m(I)}\left[\frac{2 c}{3}-\frac{d^{2}}{6 e}\right] \\
= & \int_{I} f(x) d x+\frac{\zeta_{2}-\zeta_{1}}{m(I)} \cdot \frac{d^{2}-4 e c}{3 e} .
\end{aligned}
$$

Since $\zeta_{1}=\frac{-d-\sqrt{d^{2}-4 e c}}{2 e}$ and $\zeta_{2}=\frac{-d+\sqrt{d^{2}-4 e c}}{2 e}, \zeta_{2}-\zeta_{1}=\frac{\sqrt{d^{2}-44 e c}}{e} \leq m(I)$. Hence $\left(d^{2}-4 e c\right)^{\frac{3}{2}}$ $\leq e^{3} m(I)^{3}$. So,

$$
\frac{\zeta_{2}-\zeta_{1}}{m(I)} \frac{d^{2}-4 e c}{3 e}=\frac{1}{m(I)} \frac{\left(d^{2}-4 e c\right)^{3 / 2}}{3 e^{2}} \leq \frac{1}{m(I)} \frac{e^{3} m(I)^{3}}{3 e^{2}}=\frac{1}{3} e m(I)^{2} .
$$

From the last equality of (5), it is easy to see that

$$
\begin{aligned}
\frac{1}{3} e m(I)^{2} & =\frac{60}{m(I)^{2}} \int_{I}(x-\tilde{x})^{2} f(x) d x-5 \int_{I} f(x) d x \\
& \leq \frac{60}{m(I)^{2}} \int_{I} \frac{1}{4} m(I)^{2} \cdot f(x) d x-5 \int_{I} f(x) d x \\
& =10 \int_{I} f(x) d x .
\end{aligned}
$$


Therefore,

$$
\int_{I}|\varphi(x)| d x \leq \int_{I} f(x) d x+10 \int_{I} f(x) d x=11 \int_{I} f(x) d x=11 \int_{I}|f(x)| d x .
$$

Secondly, assume there is only one zero of $\varphi$ in $(a, b)$, say $\zeta_{1} \in(a, b)$ and $\zeta_{2} \notin(a, b)$. In this case,

$$
\begin{aligned}
& \int_{I}|\varphi(x)| d x=\frac{1}{m(I)}\left[\int_{a}^{\zeta_{1}}\left(c+d x+e x^{2}\right) d x+\int_{b}^{\zeta_{1}}\left(c+d x+e x^{2}\right) d x\right] \\
& =\frac{1}{m(I)}\left\{\left[c\left(\zeta_{1}-a\right)+\frac{d}{2}\left(\zeta_{1}^{2}-a^{2}\right)+\frac{e}{3}\left(\zeta_{1}^{3}-a^{3}\right)\right]\right. \\
& \left.+\left[c\left(\zeta_{1}-b\right)+\frac{d}{2}\left(\zeta_{1}^{2}-b^{2}\right)+\frac{e}{3}\left(\zeta_{1}^{3}-b^{3}\right)\right]\right\} \\
& =\frac{\zeta_{1}-a}{m(I)}\left[c+\frac{d}{2}\left(\zeta_{1}+a\right)+\frac{e}{3}\left(\zeta_{1}^{2}+\zeta_{1} a+a^{2}\right)\right] \\
& =\frac{\left(b-\zeta_{1}\right)}{m(I)}\left[c+\frac{d}{2}\left(\zeta_{1}+b\right)+\frac{e}{3}\left(\zeta_{1}^{2}+\zeta_{1} b+b^{2}\right)\right] \\
& =\frac{\zeta_{1}-a}{m(I)}\left[c+\frac{d}{2}\left(\zeta_{1}+a\right)+\frac{e}{3}\left(\zeta_{1}^{2}+\zeta_{1} a+a^{2}\right)\right] \\
& +\frac{b-\zeta_{1}}{m(I)}\left[c+\frac{d}{2}\left(\zeta_{1}+b\right)+\frac{e}{3}\left(\zeta_{1}^{2}+\zeta_{1} b+b^{2}\right)\right] \\
& -\frac{2\left(b-\zeta_{1}\right)}{m(I)}\left[c+\frac{d}{2}\left(\zeta_{1}+b\right)+\frac{e}{3}\left(\zeta_{1}^{2}+\zeta_{1} b+b^{2}\right)\right] \\
& =\left[c+\tilde{x} d+\frac{e}{3}\left(a^{2}+a b+b^{2}\right)\right] \\
& -\frac{2\left(b-\zeta_{1}\right)}{m(I)}\left[c+\frac{d}{2}\left(\zeta_{1}+b\right)+\frac{e}{3}\left(\zeta_{1}^{2}+\zeta_{1} b+b^{2}\right)\right] \\
& =\int_{I} f(x) d x+\frac{2\left(\zeta_{1}-b\right)}{m(I)}\left[c+\frac{d}{2} b+\frac{d}{2} \zeta_{1}+\frac{1}{3} e \zeta_{1}^{2}+\frac{1}{3} e b \zeta_{1}+\frac{1}{3} e b^{2}\right] \\
& =\int_{I} f(x) d x+\frac{2\left(\zeta_{1}-b\right)}{m(I)}\left[\frac{1}{3}\left(e \zeta_{1}^{2}+d \zeta_{1}+c\right)+\frac{1}{3}(e b-d) \zeta_{1}\right. \\
& \left.+\frac{2}{3} c+\frac{d}{2} b+\frac{d}{2} \zeta_{1}+\frac{1}{3} e b^{2}\right] \\
& =\int_{I} f(x) d x+\frac{2\left(\zeta_{1}-b\right)}{m(I)}\left[\frac{1}{3} e b^{2}+\frac{1}{2} d b+\frac{1}{6} d \zeta_{1}+\frac{1}{3} e b \zeta_{1}+\frac{2}{3} c\right] \\
& =\int_{I} f(x) d x+\frac{\left(\zeta_{1}-b\right)}{3 m(I)}\left[2 e b^{2}+3 d b+d \zeta_{1}+2 e b \zeta_{1}+4 c\right] \\
& =\int_{I} f(x) d x+\frac{\left(\zeta_{1}-b\right)}{3 m(I)}\left[2\left(e \zeta_{1}^{2}+d \zeta_{1}+c\right)+2 e\left(b^{2}-\zeta_{1}^{2}\right)\right. \\
& \left.+2 d\left(b-\zeta_{1}\right)+d\left(b+\zeta_{1}\right)+2 e b \zeta_{1}+2 c\right] \\
& =\int_{I} f(x) d x+\frac{\zeta_{1}-b}{3 m(I)}\left[2 e\left(b^{2}-\zeta_{1}^{2}\right)+d\left(b-\zeta_{1}\right)+2\left(d b+e b \zeta_{1}+c\right)\right] \\
& =\int_{I} f(x) d x+\frac{\zeta_{1}-b}{3 m(I)}\left[2 e\left(b^{2}-\zeta_{1}^{2}\right)+d\left(b-\zeta_{1}\right)+2\left(b-\zeta_{1}\right)\left(e \zeta_{1}+d\right)\right] \\
& =\int_{I} f(x) d x-\frac{\left(b-\zeta_{1}\right)^{2}}{3 m(I)}\left[2 e\left(b+\zeta_{1}\right)+d+2\left(e \zeta_{1}+d\right)\right] \\
& =\int_{I} f(x) d x-\frac{\left(b-\zeta_{1}\right)^{2}}{3 m(I)}\left[4 e\left(\zeta_{1}-b\right)+3(2 e b+d)\right]
\end{aligned}
$$


From (5) we have

$$
\begin{aligned}
2 e b+d= & \frac{360 b}{m(I)^{4}} \int_{I}(x-\tilde{x})^{2} f(x) d x-\frac{30 b}{m(I)^{2}} \int_{I} f(x) d x \\
& +\frac{12}{m(I)^{2}} \int_{I} x f(x) d x+\frac{18 \tilde{x}}{m(I)^{2}} \int_{I} f(x) d x-\frac{360 \tilde{x}}{m(I)^{4}} \int_{I}(x-\tilde{x})^{2} f(x) d x \\
= & \frac{180}{m(I)^{3}} \int_{I}(x-\tilde{x})^{2} f(x) d x-\frac{12 b}{m(I)^{2}} \int_{I} f(x) d x-\frac{18 b}{m(I)^{2}} \int_{I} f(x) d x \\
& +\frac{12}{m(I)^{2}} \int_{I} x f(x) d x+\frac{18 \tilde{x}}{m(I)^{2}} \int_{I} f(x) d x \\
= & \frac{180}{m(I)^{3}} \int_{I}(x-\tilde{x})^{2} f(x) d x-\frac{12}{m(I)^{2}} \int_{I}(b-x) f(x) d x-\frac{9}{m(I)} \int_{I} f(x) d x .
\end{aligned}
$$

Hence,

$$
\begin{aligned}
\int_{I}|\varphi(x)| d x= & \int_{I} f(x) d x+\frac{4}{3} e \frac{\left(b-\zeta_{1}\right)^{3}}{m(I)}-\frac{\left(b-\zeta_{1}\right)^{2}}{m(I)} \\
& \cdot\left[\frac{180}{m(I)^{3}} \int_{I}(x-\tilde{x})^{2} f(x) d x-\frac{12}{m(I)^{2}} \int_{I}(b-x) f(x) d x\right. \\
& \left.\quad-\frac{9}{m(I)} \int_{I} f(x) d x\right] \\
\leq & \int_{I} f(x) d x+\frac{4}{3} e m(I)^{2}+\frac{12}{m(I)} \int_{I}\left(b-\zeta_{1}\right) f(x) d x+9 \int_{I} f(x) d x \\
\leq & {[1+4 \cdot 10+12+9] \int_{I} f(x) d x=62 \int_{I} f(x) d x } \\
= & 62 \int_{I}|f(x)| d x .
\end{aligned}
$$

Therefore for $f \geq 0,\|\varphi\| \leq 62\|f\|$. For general $f \in L^{1}(0,1)$, consider $f^{+}$and $f^{-}$, respectively, the same inequality can be achieved. (Q.E.D.)

LEMMA 4.2. For any $f \in L^{1}(0,1)$, if mesh $\left(\Delta_{n}\right) \rightarrow 0$, then

$$
Q_{n} f \rightarrow f \quad \text { under the } \quad L^{1}-\text { norm. }
$$

PROOF. First assume $f \in L^{2}(0,1) \subset L^{1}(0,1)$. From the way $\dot{Q}_{n} f$ is defined, obviously $\left\|f-Q_{n} f\right\|_{2}=\min \left\{\|f-g\|_{2}: g \in \Delta_{n}\right\}$ where $\|\cdot\|_{2}$ is the $L^{2}$-norm. Hence, when mesh $\left(\Delta_{n}\right) \rightarrow 0,\left\|f-Q_{n} f\right\|_{2} \rightarrow 0$, and the Cauchy inequality $\left\|Q_{n} f-f\right\| \leq\left\|Q_{n} f-f\right\|_{2}$ gives $\left\|Q_{n} f\right\| \rightarrow 0$.

Now for $f \in L^{1}(0,1)$ and $\varepsilon>0$, there exists $g \in L^{2}(0,1)$ such that $\|f-g\|<\varepsilon$. From

$$
\begin{gathered}
\left\|Q_{n} f-f\right\| \leq\left\|Q_{n} f-Q_{n} g\right\|+\left\|Q_{n} g-g\right\|+\|g-f\| \\
\leq 62\|f-g\|+\left\|Q_{n} g\right\|+\|g-f\|
\end{gathered}
$$

and $\left\|Q_{n} g-g\right\| \rightarrow 0$ we obtain $\left\|Q_{n} f-f\right\| \rightarrow 0$. (Q.E.D.)

LEMMA 4.3. If $f \in L^{1}(0,1)$ is of bounded variation, then for all $n, \vee_{0}^{1} Q_{n} f \leq 121 \vee_{0}^{1} f$.

PROOF. Since $Q_{n} f$ is piecewise quadratic,

$$
\begin{aligned}
\underset{0}{1} Q_{n} f & =\underset{0}{1} \sum_{i=1}^{n}\left(c_{i}+d_{i} x+e_{i} x^{2}\right) 1_{i} \\
& =\sum_{i=1}^{n} \frac{1}{m\left(I_{i}\right)} \int_{I_{i}}\left|d_{i}+2 e_{i} x\right| d x
\end{aligned}
$$




$$
\begin{aligned}
& +\sum_{i=1}^{n-1}\left|\frac{c_{i}+d_{i} x_{1}+e_{1} x_{i}^{2}}{m\left(I_{i}\right)}-\frac{c_{i+1}+d_{1+1} x_{1}+e_{i+1} x_{i}^{2}}{m\left(I_{1}+1\right)}\right| \\
& =\sum_{i=1}^{n} \frac{1}{m\left(I_{2}\right)} \int_{I_{i}}\left|d_{i}+2 e_{i} x\right| d x \\
& +\sum_{i=1}^{n-1} \mid\left(\frac{c_{1}}{m\left(I_{i}\right)}-\frac{c_{i+1}}{m\left(I_{i+1}\right)}\right)+\left(\frac{d_{i}}{m\left(I_{i}\right)}-\frac{d_{i+1}}{m\left(I_{i+1}\right)}\right) x_{i} \\
& +\left(\frac{e_{1}}{m\left(I_{1}\right)}-\frac{e_{i+1}}{m\left(I_{1+1}\right)}\right) x_{1}^{2} \\
& =\sum_{i=1}^{n} \frac{1}{m\left(I_{i}\right)} \int_{I_{i}}\left|d_{i}+2 e_{i} x\right| d x \\
& +\sum_{i=1}^{n-1} \mid \frac{1}{m\left(I_{i+1}\right)^{3}} \int I_{i+1}\left[12 \tilde{x}_{\imath+1} x+3 x_{i+1} x_{i}-12 x_{1}\left(x+\frac{3}{2} \tilde{x}_{i+1}\right)\right. \\
& \left.+15 x_{t}^{2}\right] f(x) d x \\
& -\frac{1}{m\left(I_{1}\right)^{3}} \int_{I_{1}}\left[12 \tilde{x}_{2} x+3 x_{\imath} x_{1-1}-12 x_{1}\left(x+\frac{3}{2} \tilde{x}_{1}\right)+15 x_{2}^{2}\right] f(x) d x \\
& +\frac{1}{m\left(I_{\imath+1}\right)^{5}} \int I_{\imath+1}\left[360 x_{\imath} \tilde{x}_{\imath+1}-60\left(2 \tilde{x}_{\imath+1}^{2}+x_{\imath+1} x_{\imath}\right)-180 x_{\imath}^{2}\right] \\
& \cdot\left(x-\tilde{x}_{\imath}+1\right)^{2} f(x) d x \\
& -\frac{1}{m\left(I_{i}\right)^{5}} \int_{I_{i}}\left[360 x_{i} \tilde{x}_{i}-60\left(2 \tilde{x}_{i}^{2}+x_{i} x_{\imath-1}\right)-180 x_{i}^{2}\right]\left(x-\tilde{x}_{z}\right)^{2} f(x) d x \\
& +\frac{3}{2}\left(\frac{1}{m\left(I_{\imath}\right)} \int_{I_{i}} f(x) d x-\frac{1}{m\left(I_{t+1}\right)} \int_{I_{i+1}} f(x) d x\right) \\
& =\sum_{i=1}^{m} \frac{1}{m\left(I_{i}\right)}\left|d_{i}+2 e_{i} x\right|+\sum_{i=1}^{n-1} \mid \frac{6}{m\left(I_{i+1}\right)^{2}} \int_{I_{i+1}}\left(x-\tilde{x}_{i}\right) f(x) d x \\
& +\frac{6}{m\left(I_{i}\right)^{2}} \int_{I_{i}}\left(x-\tilde{x}_{i}\right) f(x) d x+\frac{30}{m\left(I_{i}\right)^{3}} \int_{I_{3}}\left(x-\tilde{x}_{i}\right)^{2} f(x) d x \\
& -\frac{30}{m\left(I_{i+1}\right)^{3}} \int_{I_{i+1}}\left(x-\tilde{x}_{i+1}\right)^{2} f(x) d x+\frac{3}{2} \frac{1}{m\left(I_{i}\right)} \int_{I_{i}} f(x) d x \\
& -\frac{3}{2} \frac{1}{m\left(I_{i+1}\right)} \int_{I_{i+1}} f(x) d x
\end{aligned}
$$

Let $\Omega_{i}=\left\{(x, t): x \in I_{2}, x_{i-1} \leq t \leq x\right\}, V_{i}=\left\{(x, t, x): x \in I_{z} x_{i-1} \leq t \leq x, x_{i-1} \leq x \leq t\right\}$. Again, using the integration by parts formula for functions of bounded variation yields

$$
\begin{aligned}
& \frac{1}{m\left(I_{2+1}\right)^{2}} \int_{I_{1+1}}\left(x-x_{\imath}\right) f(x) d x=\frac{1}{m\left(I_{t+1}\right)} \int_{I_{2+1}} f(x) d x \\
& -\frac{1}{2 A\left(\Omega_{\mathrm{i}+1}\right)} \iint \Omega_{\mathrm{q}+1} f(t) d t d t, \\
& \frac{1}{m\left(I_{\mathrm{t}}\right)^{2}} \int_{I_{\mathrm{t}}}\left(x-x_{\mathrm{t}}\right) f(x) d x=-\frac{1}{2 A\left(\Omega_{\mathrm{g}}\right)} \iint \Omega_{\mathrm{i}} f(t) d t d x,
\end{aligned}
$$


SOLUTIONS OF FROBENIUS-PERRON OPERATOR EQUATIONS

481

$$
\begin{aligned}
& \frac{1}{m\left(I_{1+1}\right)^{2}} \int I_{1+1}\left(x-\dot{x}_{1+1}\right)^{2} f(x) d x=\frac{1}{4 m\left(I_{1+1}\right)} \int I_{1+1} f(x) d x \\
&-\frac{1}{2 A\left(\Omega_{1+1}\right)} \iint V_{V_{1-1}} f(t) d t d x \\
&+\frac{1}{3 V\left(V_{1+1}\right)} \iiint_{V_{1-1}} f(s) d x d t d x, \\
& \frac{1}{m\left(I_{1}\right)^{3}} \int_{I_{1}}\left(x-\tilde{x}_{1}\right)^{2} f(x) d x=\frac{1}{4 m\left(I_{1}\right)} \int_{I_{1}} f(x) d x-\frac{1}{2 A\left(\Omega_{1}\right)} \iint_{\Omega_{1}} f(t) d t d x \\
&+\frac{1}{3 V\left(V_{1}\right)} \iiint V_{1} f(s) d s d t d x,
\end{aligned}
$$

where $A\left(\Omega_{\mathrm{i}}\right)=\frac{1}{2} m\left(I_{i}\right)^{2}$ is the area of $\Omega_{\mathrm{t}}$ and $V\left(V_{\mathrm{a}}\right)=\frac{1}{6} m\left(I_{\mathrm{t}}\right)^{3}$ is the volume of $V_{1}$. Substituting into (6), we have

$$
\begin{aligned}
& \underset{0}{1} Q_{n} f=\sum_{i=1}^{n} \frac{1}{m\left(I_{i}\right)} \int I_{I_{i}}\left|d_{i}+2 e_{i} x\right| d x \\
& +\sum_{i=1}^{n-1} \mid \frac{6}{m\left(I_{i+1}\right)} \int_{I_{i+1}} f(x) d x-\frac{3}{A\left(\Omega_{i+1}\right)} \iint \Omega_{\Omega_{i+1}} f(t) d t d x \\
& -\frac{3}{A\left(\Omega_{\mathrm{i}}\right)} \iint_{\Omega_{i}} f(t) d t d x+\frac{15}{2 m\left(I_{i}\right)} \int_{I_{i}} f(x) d x \\
& -\frac{15}{A\left(\Omega_{\mathrm{t}}\right)} \iint \Omega_{\Omega_{\mathrm{t}}} f(t) d t d x+\frac{10}{V\left(V_{\mathrm{t}}\right)} \iiint v_{\mathrm{q}} f(s) d s d t d x \\
& -\frac{15}{2 m\left(I_{i+1}\right)} \int I_{i+1} f(x) d x+\frac{15}{A\left(\Omega_{1+1}\right)} \iint \Omega_{1+1} f(t) d t d x \\
& -\frac{10}{V\left(V_{i+1}\right)} \iiint V_{i+1} f(s) d s d t d x+\frac{3}{2 m\left(I_{1}\right)} \int_{I_{i}} f(x) d x \\
& -\frac{3}{2 m\left(I_{i+1}\right)} \int_{I_{i+1}} f(x) d x \\
& =\sum_{i=1}^{n} \frac{1}{m\left(I_{i}\right)} \int_{I_{i}}\left|d_{i}+2 e_{i} x\right| d x \\
& +\sum_{i=1}^{n-1} \mid \frac{9}{m\left(I_{i}\right)} \int_{I_{i}} f(x) d x-\frac{3}{2 m\left(I_{i+1}\right)} \int_{I_{i+1}} f(x) d x \\
& +\frac{12}{A\left(\Omega_{i+1}\right)} \iint \Omega_{\Omega_{i+1}} f(t) d t d x-\frac{18}{A\left(\Omega_{i}\right)} \iint \Omega_{\Omega_{i}} f(t) d t d x \\
& +\frac{10}{V\left(V_{i}\right)} \iiint v_{i} f(s) d s d t d x-\frac{10}{V\left(V_{i+1}\right)} \iiint v_{i+1} f(s) d s d t d x \mid \\
& \leq \sum_{i=1}^{n} \frac{1}{m\left(I_{i}\right)} \int_{I_{i}}\left|d_{i}+2 e_{i} x\right| d x \\
& +3 \sum_{i=1}^{n-1}\left|\frac{1}{m\left(I_{i}\right)} \int_{I_{i}} f(x) d x-\frac{1}{m\left(I_{i}+1\right)} \int_{I_{i+1}} f(x) d x\right| \\
& +6 \sum_{i=1}^{n-1}\left|\frac{1}{m\left(I_{i}\right)} \int_{I_{i}} f(x) d x-\frac{1}{A\left(\Omega_{i}\right)} \iint \Omega_{i} f(t) d t d x\right| \\
& +12 \sum_{i=1}^{n-1}\left|\frac{1}{A\left(\Omega_{i+1}\right)} \iint \Omega_{i+1} f(t) d t d x-\frac{1}{A\left(\Omega_{i}\right)} \iint \Omega_{i} f(t) d t d x\right|
\end{aligned}
$$




$$
\begin{aligned}
& +10 \sum_{i=1}^{n-1} \mid \frac{1}{V\left(V_{i}\right)} \iiint v_{i} f(s) d s d t d x \\
& -\frac{1}{V\left(V_{1+1}\right)} \iiint v_{1+1} f(s) d s d t d x \\
& \leq \sum_{i=1}^{n} \frac{1}{m\left(I_{i}\right)} \int_{I_{i}}\left|d_{i}+2 e_{i} x\right| d x \\
& +3 \underset{0}{\stackrel{1}{V}} f+6 \underset{0}{\stackrel{1}{\vee}} f+12 \underset{0}{\vee} f+10 \underset{0}{\vee} f \\
& =\sum_{i=1}^{n} \frac{1}{m\left(I_{i}\right)} \int_{I_{i}}\left|d_{i}+2 e_{i} x\right| d x+31 \underset{0}{\stackrel{1}{v} f .}
\end{aligned}
$$

Let's estimate the first term of (7). For $i=1, \ldots, n, \frac{1}{m\left(I_{2}\right)} \int_{I_{1}}\left|d_{1}+2 e_{1} x\right| d x$ is the variation of $Q_{n} f$ on $I_{i}$. For simplicity, we omit the subscript. Let $\varphi(x)=\frac{1}{m(I)}\left(c+d x+e x^{2}\right), I=[a, b]$. Without loss of generality, assume $e>0$. Then $\zeta=-\frac{d}{2 e}$ is the minimal point of $\varphi$ and $\varphi\left(-\frac{d}{2 e}\right)=\frac{1}{m(I)}\left(c-\frac{d^{2}}{4 e}\right)$ is the minimal value of $\varphi$. If $a<\zeta<b$, then

$$
\begin{aligned}
\underset{I}{\vee \varphi} & =\frac{1}{m(I)}(\varphi(a)+\varphi(b)-2 \varphi(\zeta)) \\
& =\frac{1}{m(I)}\left(c+d a+e a^{2}+c+d b+e b^{2}-2 c+\frac{d^{2}}{2 e}\right) \\
& =\frac{1}{m(I)}\left[d(a+b)+e\left(a^{2}+b^{2}\right)+\frac{d^{2}}{2 e}\right] \\
& =\left[-2 e \zeta(a+b)+e\left(a^{2}+b^{2}\right)+2 e \zeta^{2}\right] / m(I) \\
& =e\left[2 \zeta^{2}-2 \zeta(a+b)+a^{2}+b^{2}\right] / m(I) \\
& =e\left[(a-b)^{2}+2 a b-2 \zeta(a+b)+2 \zeta^{2}\right] / m(I) \\
& =\frac{1}{m(I)}\left[e m(I)^{2}+2 e(a-\zeta)(b-\zeta)\right] \leq e m(I) \\
& =\frac{15}{m(I)}\left[\frac{12}{m(I)^{2}} \int_{I}(x-\tilde{x})^{2} f(x) d x-\int_{I} f(x) d x\right] \\
& \leq 30\left|\frac{1}{m(I)} \int_{I} f(x) d x-\frac{3}{A(\Omega)} \iint_{\Omega} f(t) d t d x+\frac{2}{V(V)} \iint_{V} f(s) d s d t d x\right| \\
& \leq 90 V_{I} f .
\end{aligned}
$$

If $\zeta \notin(a, b)$, then

$$
\begin{aligned}
\underset{I}{\vee} \varphi & =\frac{1}{m(I)}|\varphi(a)=\varphi(b)|=\frac{1}{m(I)}\left|d(b-a)+e\left(b^{2}-a^{2}\right)\right| \\
& =|d+2 e \tilde{x}|=\frac{12}{m(I)}\left|\int_{I}(x-\tilde{x}) f(x) d x\right| \\
& \leq 6\left|\frac{1}{m(I)} \int_{I} f(x) d x-\frac{1}{A(\Omega)} \iint_{\Omega} f(t) d t d x\right| \\
& \leq 6 \underset{I}{\vee} f<90 \underset{I}{\vee} f .
\end{aligned}
$$

Substituting into (7), we have

$$
{\underset{0}{V}}_{0}^{1} Q_{n} f \leq 90 \sum_{i=1}^{n} \underset{I}{\vee} f+31 \underset{0}{1} f=121 \underset{0}{\vee} f .
$$


LEMMA 4.4. Let $P_{n}=\left.Q_{n} \circ P_{S}\right|_{\Delta_{n}}$, where $P_{S}$ is the Frobenius-Perron operator associated with $S:[0,1] \rightarrow[0,1]$. Then $P_{n}$ has a nontrivial fixed point in $\Delta_{n}$.

PROOF. Denote by $\tilde{P}_{n}$ the representations of $P_{n}: \Delta_{n} \rightarrow \Delta_{n}$ under the basis $\left\{1_{i}, x 1_{1}, x^{2} 1_{i}\right\}_{i=1}^{n}$. Let $\zeta=\left(1, \tilde{x}_{1}, \tilde{y}_{1}, 1, \tilde{x}_{2}, \tilde{y}_{2}, \cdots, 1, \tilde{x}_{n}, \tilde{y}_{n}\right)$ where $\tilde{y}_{i}=\frac{1}{3}\left(x_{1}^{2}+x_{i} x_{i-1}+x_{i-1}^{2}\right)$. Then for $i=1, \ldots, n$,

$$
\begin{aligned}
\left(\zeta \tilde{P}_{n}\right)_{3(i-1)+1} & =\sum_{j=1}^{n}\left(c j\left(1_{i}\right)+\tilde{x}_{j} d_{j}\left(1_{i}\right)+\tilde{y}_{j} e_{j}\left(1_{j}\right)\right) \\
& =\sum_{j=1}^{n} \int I_{j}\left(P_{S} 1_{i}\right)(x) d x=\int_{0}^{1}\left(P_{S} 1_{i}\right)(x) d x \\
& =\int_{0}^{1} 1_{i}(x) d x=1, \\
\left(\zeta \tilde{P}_{n}\right)_{3(1-1)+2} & =\sum_{j=1}^{n}\left(c_{j}\left(x 2_{i}\right)+\tilde{x}_{j} d_{j}\left(x 1_{i}\right)+\tilde{y}_{j} e_{j}\left(x 1_{i}\right)\right) \\
& =\sum_{j=1}^{n} \int{ }_{I}\left(P_{S}\left(x 1_{i}\right)\right)(x) d x=\int_{0}^{1}\left(P_{S}\left(x 1_{i}\right)\right)(x) d x \\
& =\int_{0}^{1}{ }_{0} 1_{j}(x) d x=\tilde{x}_{j}, \\
\left(\zeta \tilde{P}_{n}\right)_{3 i} & =\sum_{j=1}^{n}\left(c,\left(x^{2} 1_{i}\right)+\tilde{x}_{j} d_{j}\left(x^{2} 1_{i}\right)+\tilde{y}_{j} e_{j}\left(x^{2} 1_{i}\right)\right) \\
& =\sum_{j=1}^{n} \int I_{j}\left(P_{S}\left(x^{2} 1_{i}\right)\right)(x) d x=\int_{0}^{1}\left(P_{S}\left(x^{2} 1_{i}\right)\right)(x) d x \\
& =\int_{0}^{1} x^{2} 1_{i}(x) d x=\tilde{y}_{i} .
\end{aligned}
$$

That is, $\zeta$ is a left eigenvector of the matrix $\tilde{P}_{n}$ corresponding to the eigenvalue 1 . Therefore, there is a nonzero $c \in \Re^{3 n}$ such that $\tilde{P}_{n} c=c$. Thus $P_{n}$ has a nonzero fixed point in $\Delta_{n}$. (Q.E.D.)

THEOREM 4.1. Let $S:[0,1] \rightarrow[0,1]$ be piecewise $C^{2}$ and $M=\inf |S|>242$. Let $\left\{f_{n}\right\}$ bf a sequence of fixed points of $P_{n}$ in $\Delta_{n}$ with $\left\|f_{n}\right\|=1$. Then there exists a subsequence $\left\{f_{n_{1}}\right\} \subset\left\{j_{n}\right\}$ convergent to a fixed point of $P_{S}$.

PROOF. By the Lasota-Yorke inequality in the previous section, we have for any $n$

$$
\begin{aligned}
\underset{0}{1} f_{n} & =\underset{0}{1} P_{n} f_{n}=\underset{0}{1} Q_{n} \circ P_{S} f_{n} \leq 121 \underset{0}{1} P_{S} f_{n} \\
& \leq 121\left(\alpha\left\|f_{n}\right\|+\frac{2}{M} \underset{0}{1} f_{n}\right)=121 \alpha+\beta \underset{0}{1} f_{n}
\end{aligned}
$$

with $\beta=\frac{242}{M}<1$. Hence

$$
\stackrel{1}{v} f_{n} \leq \frac{121 \alpha}{1-\beta}<+\infty
$$

From the Helly theorem there is a subsequence $\left\{f_{n_{i}}\right\} \subset\left\{f_{n}\right\}$ which converges to some $f \in L^{1}(0,1)$. From

$$
\begin{aligned}
\left\|P_{S} f-f\right\| \leq \| f & -f_{n_{i}}\|+\| f_{n_{i}}-Q_{n_{i}} \circ P_{S} f_{n_{i}} \| \\
& +\left\|Q_{n_{i}} \circ P_{S} f_{n_{i}}-Q_{n_{i}} \circ P_{S} f\right\| \\
& +\left\|Q_{n_{i}} \circ P_{S} f-P_{S} f\right\|
\end{aligned}
$$

it is obvious that $P_{S} f=f$. (Q.E.D.)

The proof of the following corollary is the same as that of Corollary 3.1. 
COROLLARY 4.1. Let $S:[0,1] \rightarrow[0,1]$ be piecewise $C^{2}$ and inf $\left|S^{\prime}\right|>1$. Then, a sequence of functions can be constructed from piecewise quadratic functions which converge to a nontrivial fixed point of $\boldsymbol{P}_{\boldsymbol{S}}$.

\section{CONCLUSIONS.}

In this paper, the piecewise linear and piecewise quadratic polynomial projection methods are proposed for the computation of invariant densities of the Frobenius-Perron operator. The convergence of the methods is proved for a class of measurable nonsingular transformations of the unit interval into itself which satisfy the condition of the Lasota-Yorke theorem. Our proof is based on the following observation: The projections $Q_{n}: L^{1}(0,1) \rightarrow L^{1}(0,1)$ defined in the previous sections satisfy

(1) $\left\|Q_{n}\right\| \leq M, \quad M$ is a constant.

(2) $Q_{n} f \rightarrow f$ strongly for any $f \in L^{1}(0,1)$.

(3) $\vee_{0}^{1} Q_{n} f \leq C \vee_{0}^{1} f$ for any $f \in L^{1}(0,1)$ of bounded variation, where $C$ is a constant.

(4) $Q_{n} \circ P$ has a nontrivial fixed point $f_{n}$ for each $n$.

In general, the projection method for the Frobenius-Perron operator equation $P f-f=0$ is convergent if the "discretization" operators $Q_{n}$ satisfy the above four requirements, as the following theorem shows.

THEOREM 5.1. Suppose the sequence of operators $Q_{n}$ satisfy the conditions (1) through (4) above. Then a sequence of approximate functions can be constructed which converge to a nontrivial fixed point of $P_{S}$ when $S:[0,1] \rightarrow[0,1]$ is piecewise $C^{2}$ and inf $\left|S^{\prime}\right|>1$.

The proof of this theorem follows exactly the same line of arguments in Theorem 3.1 and Theorem 4.1, in which $M=2, C=13$, and $M=62, C=121$, respectively.

Based on the convergence analysis of the piecewise linear and piecewise quadratic polynomial approximation methods, we believe that for general higher order piecewise polynomial projection method, the convergence can also be established, although it will be more tedious and complicated.

It is important to estimate the convergence rate for a convergent numerical method. The further research will be focused on this aspect for our projection methods for Frobenius-P.rron operator equation or more general Markov operator equation. For solving noncompact op "ator equations in nonreflexive Banach spaces, this field is not fully developed, although is essent 1.1 to physical sciences.

ACKNOWLEDGEMENT. This research was supported in part by NSF under Grant DMS8902663.

\section{REFERENCES}

1. KOHDA, T. and MURAO, K., Elec. and Commu. in Japan 65-A 6 (1982) 1.

2. LASOTA, A. and MACKEY, M.C., Probabilistic Properties of Deterministic Systems, (Cambridge, 1985).

3. LASOTA, A. and YORKE, J.A., Trans. Amer. Math. Soc. 186 (1973) 481.

4. LI, T.Y., J. Approx. Theory 17 (1976) 177.

5. LI, T.Y. and YORKE, J.A., Trans. Amer. Math. Soc. 235 (1978) 183.

6. MIKHLIN, S.G. and SMOLITSKIY, L., Approximate Methods for Solution of Differential and Integral Equations, (American Elsevier Publishing Company, 1967) 253.

7. NATANSON, L.P., Theory and Functions of Real Variable, (Ungar, New York, 1961).

8. ULAM, S.M. A collection of mathematical problems, Interscience Tracts in Pure and Applied Math. 8, (Interscience, New York, 1960). 


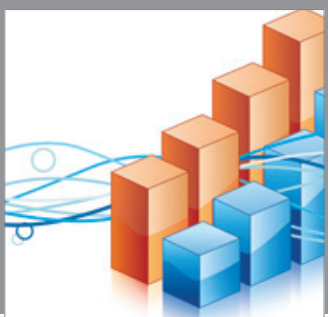

Advances in

Operations Research

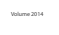

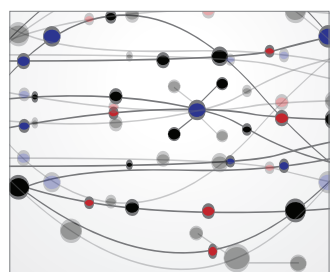

\section{The Scientific} World Journal
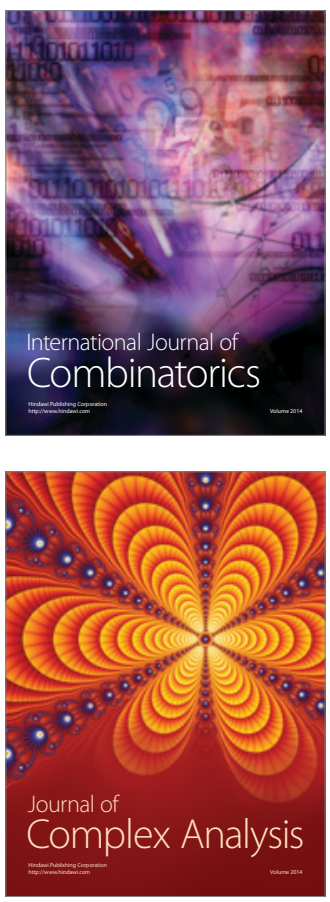

International Journal of

Mathematics and

Mathematical

Sciences
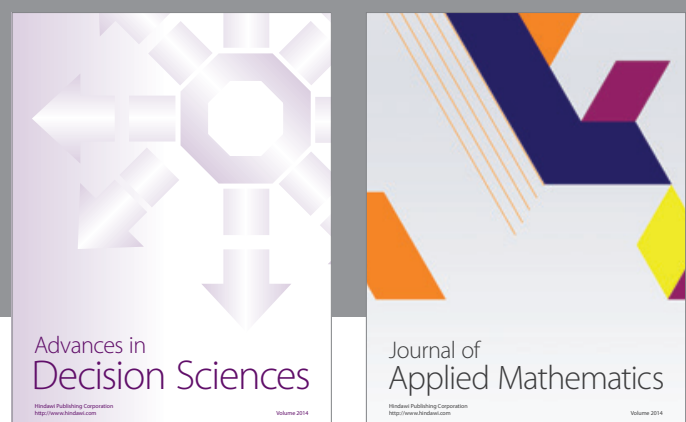

Journal of

Applied Mathematics
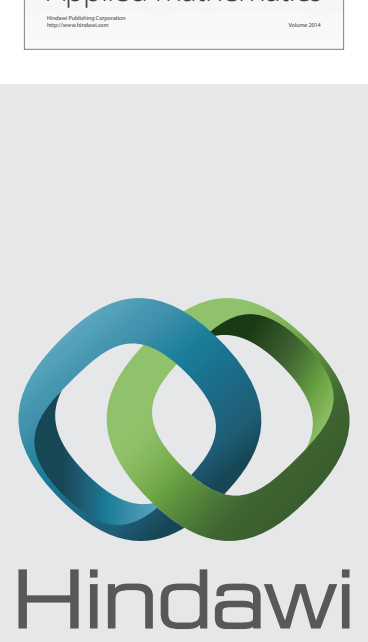

Submit your manuscripts at http://www.hindawi.com
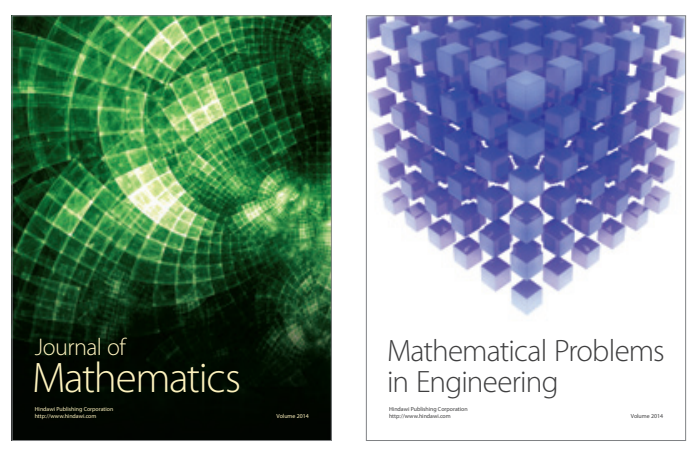

Mathematical Problems in Engineering
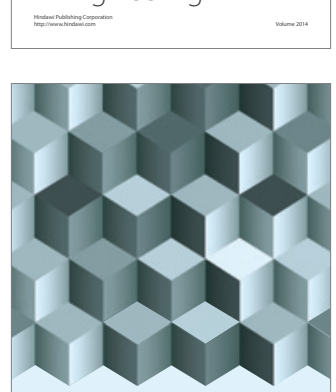

Journal of

Function Spaces
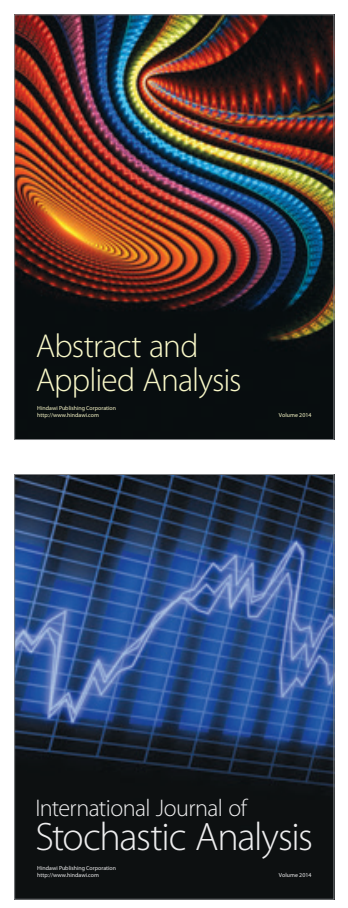

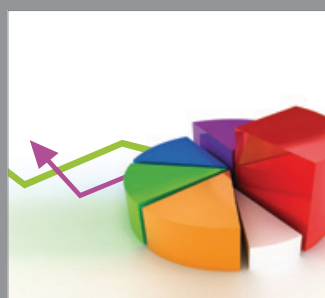

ournal of

Probability and Statistics

Promensencen
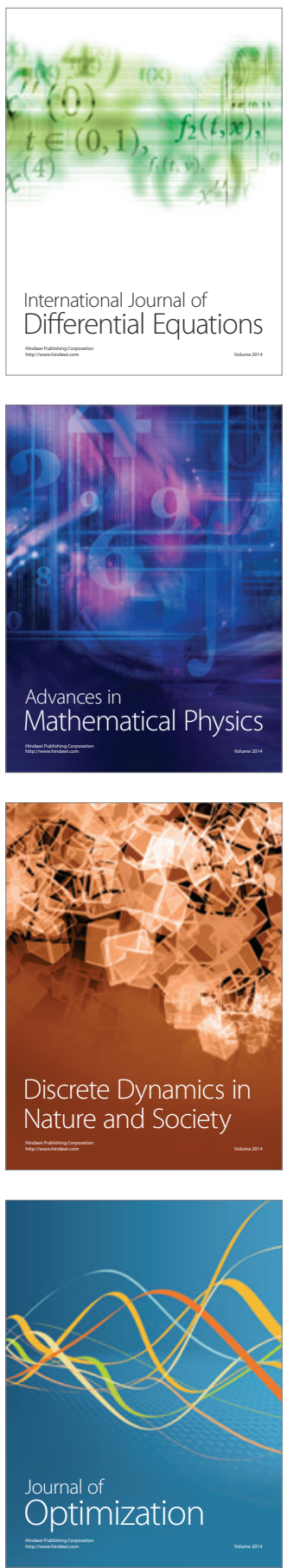